\title{
Exploiting Anisotropy for In Situ Measurement of Silicon Etch Rates in KOH Solution
}

\author{
Harold G. G. Philipsen, ${ }^{\mathrm{Z}}$ Niek J. Smeenk, Hans Ligthart, and John J. Kelly* \\ Debye Institute, Condensed Matter and Interfaces, Utrecht University, 3508 TA Utrecht, \\ The Netherlands
}

Anisotropic etching of V-grooves in a masked substrate provides the basis for two simple methods for in situ measurement of etch rates of $\mathrm{Si}(100)$. The width of the (100) facet defining the base of the groove and thus its surface area decreases at a rate which is determined by the etch rate in the (100) direction. By measuring voltammograms at regular intervals during etching we were able to monitor the change in geometry of the groove. In a complementary approach, in situ optical microscopy was used for determining etch rates in real time. An application of the method is described.

(C) 2006 The Electrochemical Society. [DOI: 10.1149/1.2203237] All rights reserved.

Manuscript submitted January 25, 2006; revised manuscript received April 4, 2006. Available electronically May $17,2006$.

Anisotropic etching of silicon is an essential step in device fabrication. ${ }^{1,2}$ Microelectromechanical systems (MEMS) and optoelectronics rely on the characteristic forms that result from the differences in etch rate of different crystallographic orientations. ${ }^{3-5}$ It is therefore essential to be able to measure etch rates accurately and to detect any changes that may occur in these rates during a fabrication process. In addition, it may be necessary to develop a new etchant or to adapt an existing one, e.g., to determine the influence of an additive in a wide concentration range or to investigate the effect of temperature or of a shift in potential of the sample on the dissolution rates.

Methods based on the etching of wagon-wheel patterns ${ }^{6-8}$ or of semiconductor (hemi) spheres ${ }^{9-11}$ have the advantage of yielding dissolution rates for a whole range of orientations in a single experiment. These methods have a number of disadvantages: they are not simple, analysis of the results is cumbersome and, most importantly, the etching conditions generally differ from those used for device fabrication, e.g., sample geometry, hydrodynamics, gas evolution and galvanic interaction between facets may be important in determining etch rate and morphology. ${ }^{12,13}$

At the other extreme, one can measure etch rates simply by masking a wafer of a given orientation and, after etching, determining the etched depth with a profilometer. In addition, examination of the cleaved sample with optical microscopy or scanning electron microscopy (SEM) can give information about etch rates of other orientations (via the underetching ${ }^{14}$ ). Because each etch rate requires a number of separate experiments this approach is tedious, especially if one wishes to screen a wide range of experimental conditions. Glembocki and coworkers adapted this approach by using strips of silicon masked with an oxide layer containing square windows. ${ }^{15}$ By retracting the sample stepwise from the solution they were able to vary the time that windows were exposed to the etchant and were therefore able to measure a range of etch rates (in their case as a function of potential) in a single experiment. A disadvantage of this approach is that etching close to the solution/air boundary is important. Conditions near this boundary are obviously not the same as in a typical etching experiment, in particular because gas is being evolved. In addition, quenching of the etching reaction above the solution after a retraction step is not well defined.

The methods described so far can be classed as ex situ: the rate is determined after removal of the sample from the solution. Interferometry, on the other hand, offers the possibility of in situ monitoring of etch rates in real time. Tosaka et al. demonstrated this by measuring transmission and reflection spectra during the formation of membranes. ${ }^{16}$ The interference pattern in such a plan-parallel geometry depends on the thickness of the sample, which changes continuously as the material dissolves. Therefore, the intensity of the trans-

\footnotetext{
* Electrochemical Society Active Member.

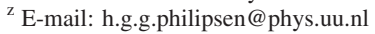

mitted or reflected light is modulated over time with a period that correlates with the etch rate. With a comparable setup Steinsland et al. studied etching of the three important crystallographic orientations of silicon, i.e., (100), (110), and (111), and developed a simulation model. ${ }^{17}$ Surface roughness and gas evolution can be a problem with this approach.

In this paper we show how one can exploit the anisotropy of silicon etching to develop two rather simple methods for in situ measurement of the etch rate of certain faces of the semiconductor. With an aligned mask V-grooves defined by (111) facets are etched in a (100) substrate. ${ }^{12,14,18}$ Because the electrochemistry of the two faces is also strongly anisotropic, ${ }^{19,20}$ in situ anodic current measurements can be used to determine accurately the change in geometry of the grooves as a result of etching. In a second but complementary method optical microscopy combined with a flow cell ${ }^{21}$ is used to map the sample geometry, which also allows a continuous determination of etch rates.

\section{Principle of Approach}

Because the etch rate of the (111) face of silicon in $\mathrm{KOH}$ solution is much lower than that of other faces, ${ }^{4,6,13,14,22}$ etching through windows in an aligned mask pattern on a silicon (100) substrate gives well-defined (111) facets. ${ }^{13,14,18,19,22,23}$ V-grooves and inverted pyramids are formed in the case of rectangular and square mask openings, respectively. ${ }^{1,13,20}$ During the process, the silicon is etched chemically at open-circuit potential and slow-etching (111) facets

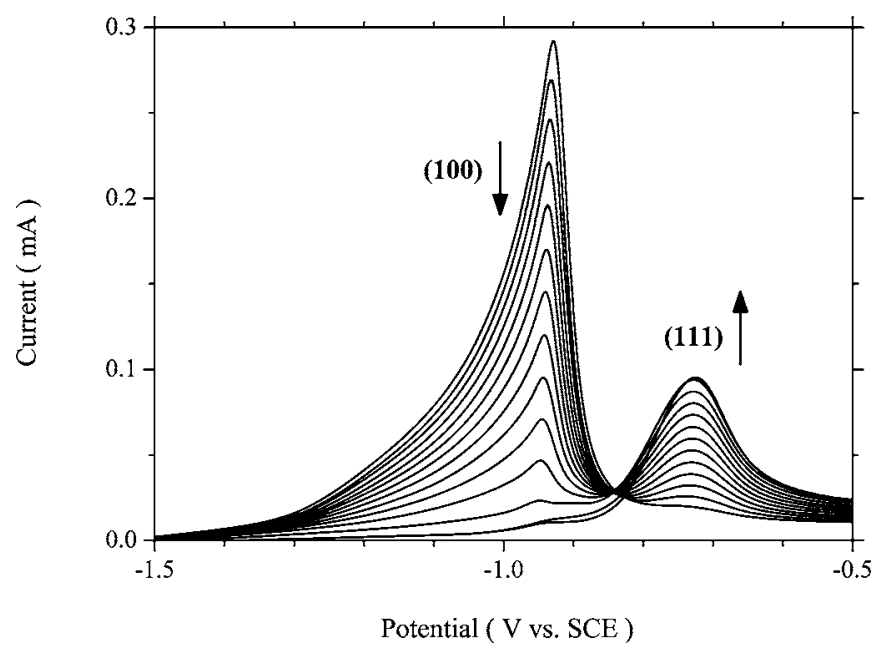

Figure 1. Voltammograms measured at $20 \mathrm{~min}$ intervals during V-groove formation in p-type $\mathrm{Si}$ in $5.0 \mathrm{M} \mathrm{KOH}$ solution at $60.0^{\circ} \mathrm{C}$. Width of mask window: $100 \mu \mathrm{m}$ (sample type B). The potential was scanned from -2.00 to $+0.50 \mathrm{~V}$ at a rate of $25 \mathrm{mV} / \mathrm{s}$. 
become exposed to the $\mathrm{KOH}$ solution. Figure 1 shows how the electrochemistry of a p-type wafer etching in $5.0 \mathrm{M} \mathrm{KOH}$ solution at $60.0^{\circ} \mathrm{C}$ changes as the V-groove is formed. For these experiments anisotropic etching at open-circuit potential was interrupted at regular intervals (20 min) and a voltammogram was measured: the potential was scanned to a positive value and the current was recorded. Subsequently the applied potential was removed and the system returned to open-circuit condition. The voltammograms show two current peaks, due to the (100) and (111) faces; anodic oxidation and passivation of the (111) sidewalls occur at considerably more positive potential. During anisotropic etching, the (100) surface area decreases continuously with time and the (111) surface area increases with time. After the grooves are fully formed, only (111) surfaces are exposed to the etchant and the peak current remains constant. A detailed discussion on the mechanism of anisotropic etching and the electrochemistry of the two crystal planes can be found elsewhere. ${ }^{19}$

By measuring the peak currents taken from voltammograms at various stages of $\mathrm{V}$-groove formation we can determine the relative areas of the faces and thus the geometry of the groove; this allows us to follow the (100) etch rate. If etching is carried out at potentials positive with respect to the open-circuit value, i.e., in the anodic range, the etch rate can be determined simply by measuring the current as a function of time at that potential. Alternatively, the progress of V-groove formation can be followed directly in a flow cell by means of an optical microscope.

\section{Experimental}

For the experiments, 4 in. (100)-oriented wafers were used. On these Czochralski grown n-type (phosphorus doped, $1-10 \Omega \mathrm{cm}$, Okmetic) and p-type (boron doped, 5-10 $\Omega \mathrm{cm}$, Okmetic) substrates a $100 \mathrm{~nm}$ thick silicon-rich nitride masking layer (SiRN) was deposited by LPCVD. Windows were opened in the nitride layer by a plasma etch (DRIE). By using two photolithography steps, the edges of the windows were aligned along the $\langle 110\rangle$ direction with an accuracy of $0.05^{\circ} .^{23}$

After preparation, the wafers were diced into samples of 20 $\times 20 \mathrm{~mm}$. Different types of electrode were prepared for these experiments. To ensure a significant current in the electrochemical measurements each sample contained an array of openings. For studying V-groove formation, the A samples consisted of 210 rectangular mask openings $(35 \times 6$ array, $100 \mu \mathrm{m}$ width, and $1.0 \mathrm{~mm}$ length). The $\mathrm{B}$ samples contained arrays of rectangular openings of different widths $(100,150$, and $200 \mu \mathrm{m}, 8.0 \mathrm{~mm}$ length, and number of openings: 30,25 , and 20 ). Details regarding preparation and cleaning of the samples can be found in Ref. 19.

For the electrochemical measurements, the configuration consisted of three electrodes. The silicon working electrode, a platinum counter electrode and a saturated calomel reference electrode (SCE) were connected to a potentiostat (EG\&G Princeton Applied Research, PAR-273-A) which was computer controlled by LabView. All measurements were performed in the dark and the etchant was continuously purged with argon to remove dissolved gases.

In a series of complementary experiments, measurements were performed with a flow cell, ${ }^{21}$ which allowed us to measure groove dimensions in situ with an optical microscope. Using a long working distance objective lens (Nikon, 20x, working distance $24.0 \mathrm{~mm}$, CFI L Plan Epi SLWD) on the microscope (Zeiss, Axioplan 2) equipped with a CCD camera, it was possible to view the etching sample via a quartz window at the top of the cell. The flow cell was made from Teflon and Kel-F and the thickness of the liquid layer over the sample surface was $6.0 \mathrm{~mm}$.

To provide a reference for the methods used in this work, samples were etched for various times under open-circuit conditions in $5.0 \mathrm{M} \mathrm{KOH}$ at $60.0^{\circ} \mathrm{C}$. The dimensions of the V-grooves were determined with SEM after cleaving of the single crystalline material. In Fig. 2, the widths of the (100) and (111) facets are plotted as a function of etching time. The linear change in surface areas shows that the (100) etch rate is constant. From the extrapolation of the

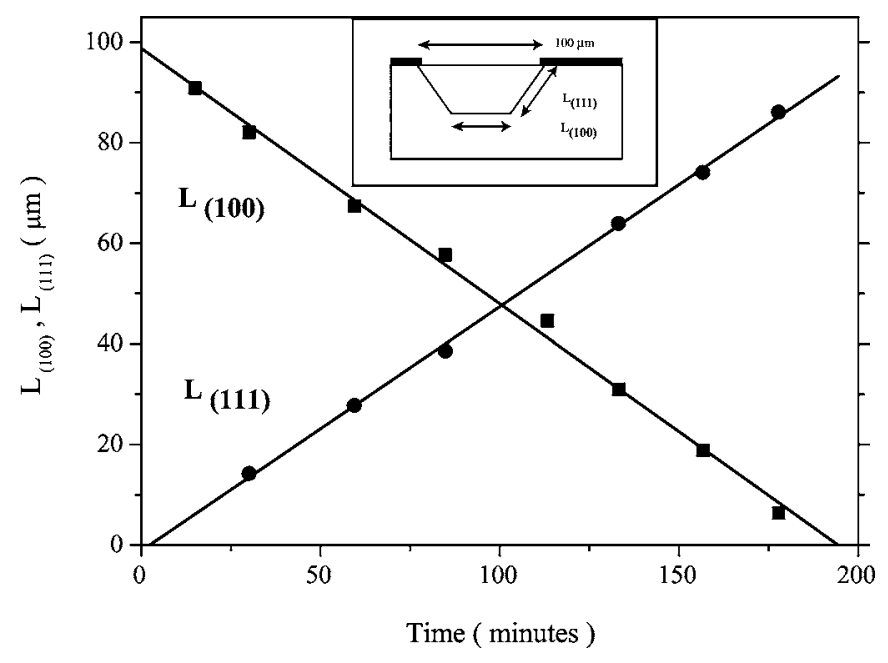

Figure 2. Formation of $100 \mu \mathrm{m}$ wide V-grooves under open-circuit conditions (n-type $\mathrm{Si}$, sample type A). After cleaving, dimensions of the V-grooves were measured by SEM. The lines are linear fits through the data points. Definitions of $\mathrm{L}_{(100)}$ and $\mathrm{L}_{(111)}$ are shown in the inset of the figure. Conditions: $5.0 \mathrm{M} \mathrm{KOH}$ at $60.0^{\circ} \mathrm{C}$.

linear fit of the trace for $\mathrm{L}_{(100)}$ the end point of the etch and the (100) etch rate can be calculated $(21.8 \mu \mathrm{m} / \mathrm{h}$ ). The anisotropic ratio (defined here as the etch rate of the (100) facet divided by the etch rate of the (111) facet) is larger than one order of magnitude, ${ }^{13}$ and effects of the mask underetch on the width of the (100) facet are negligible.

\section{Results and Discussion}

Electrochemical approach.-The results shown in Fig. 1 clearly illustrate that it is possible to monitor the etching process simply by measuring voltammograms during V-groove formation and that this can give information about both facets. Figure 3 a shows the peak current for the (100) and (111) peaks $\left(\mathrm{I}_{\text {Peak,(100) }}\right.$ and $\left.\mathrm{I}_{\text {Peak,(111) }}\right)$ vs time for the series of voltammograms shown in Fig. 1. As the (100) etch rate is constant, the (100) peak current decreases and the (111) peak current increases linearly with etching time, as expected for this geometry (see inset of Fig. 2). After the V-grooves are fully formed, the (111) peak current remains constant.

To show the influence of the dimensions, measurements were performed on arrays of 100, 150, and $200 \mu \mathrm{m}$ wide V-grooves under identical conditions. In Fig. 3b, the (100) peak current of the CV is plotted vs time with a correction for the different number of grooves in these arrays. It is expressed as the peak current per groove. The figure shows that the initial (100) peak current is linearly dependent on the surface area, i.e., the width of the mask opening. On the basis of the geometries of the electrodes and extrapolations of the linear fits of the peak current data points (see Fig. 3b), the passivation current density for the (100) facet was calculated to be $(1.61+/$ $-0.04) \mathrm{mA} / \mathrm{cm}^{2}$. This corresponds well to results reported before. ${ }^{19}$ As the rate of groove formation is the same in all cases, i.e., the (100) etch rate is constant, these peak current-time plots show parallel lines. This clearly illustrates that the (100) peak current can be used to measure the dimensions of V-grooves and thus the (100) etch rate.

For the results shown in Fig. 1, both crystal facets were passivated with anodically grown oxide after each scan. Consequently the slopes of the straight lines shown in Fig. 3 do not represent the "true" chemical etch rate of the (100) surface. Three possible effects have to be considered: $(i)$ contribution from electrochemical etching during the scan to positive potential. Previous work has shown that this "anodic" etch rate is much smaller than the chemical etch rate. ${ }^{25}$ (ii) An effect of applied potential during the scan on the chemical 

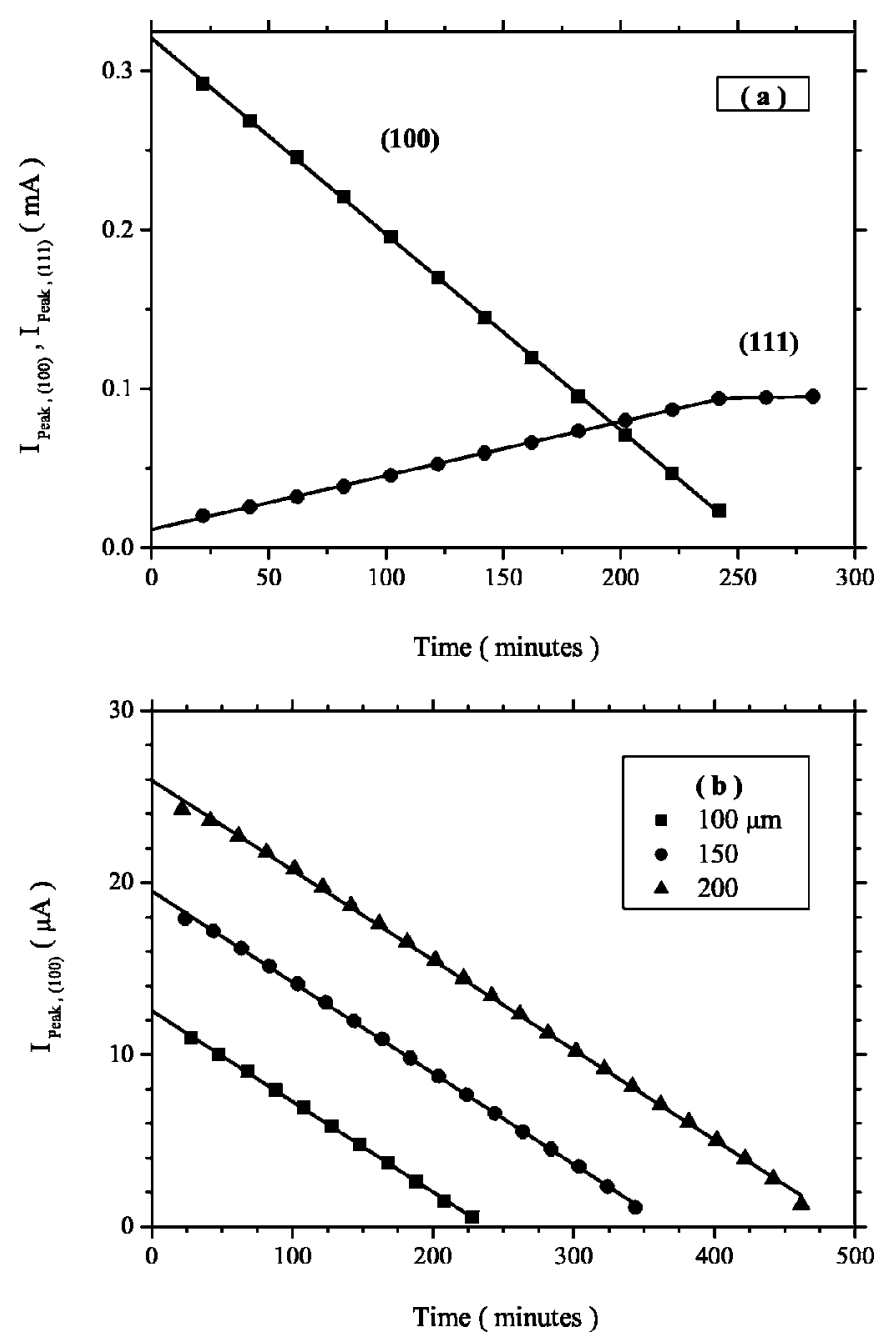

Figure 3. (a) Peak current for the (100) and (111) surfaces vs time, as determined from the series of voltammograms in Fig. 1. (b) Peak current for the (100) surface vs time for different widths of V-grooves (sample type B). Current is expressed per groove. The lines are linear fits through the data points.

etch rate. This might be important in the case of n-type Si: see below. However, the time required to scan from open-circuit to the peak potential is short $(\sim 20 \mathrm{~s})$ as compared to the interval between the measurement of two voltammograms (20 min); (iii) The formation of oxide. After each electrochemical measurement in Fig. 1 the (100) and (111) faces are passivated. Before anisotropic etching can recommence the oxide must be dissolved. Because the oxide etchback time is the same for all the runs in Fig. 1 the etch rate can be corrected for this effect.

As the peak current density is larger for the (100) plane, ${ }^{19,20,24}$ it is more accurate to use this plane for the characterization of the groove dimensions. Therefore, it suffices to end the scan at a potential slightly more positive than the (100) peak potential. This minimizes effects of the oxide etch back time and possible influence of the applied potential on the etching process. If one is interested in chemical etching at applied potentials positive with respect to the open-circuit potential, then the time-dependence of the anodic current of the (100) face can be used to measure etch rates. Like the peak current, this current decreases linearly in time and can be used to define the groove geometry.

Optical observation.-The results shown so far rely on the measurement of current in an external circuit and the dimensions of the structures are determined "indirectly" via the linear dependence of

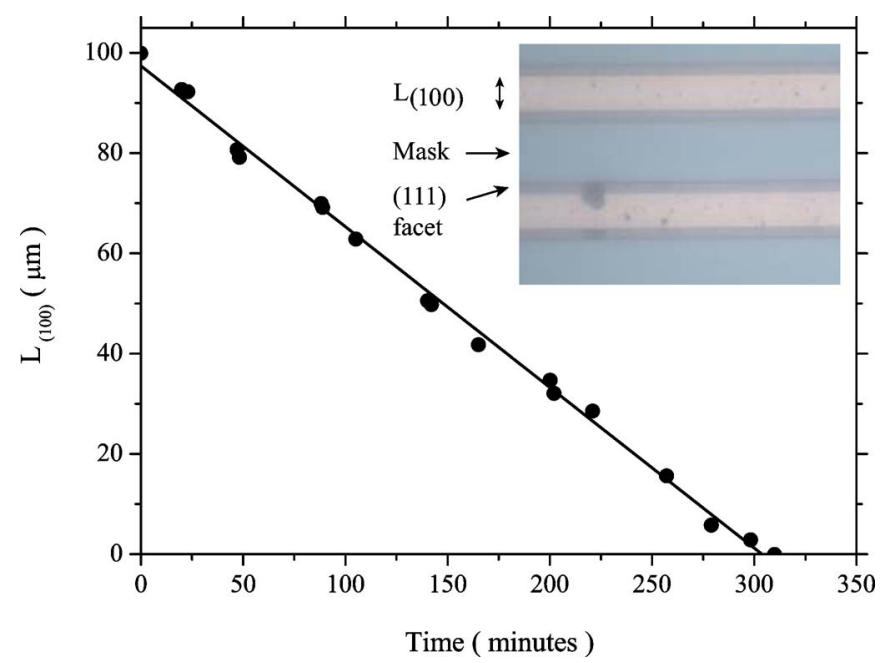

Figure 4. In situ measurement of $\mathrm{L}_{(100)}$ during V-groove formation in p-type $\mathrm{Si}$ (sample type A). Data were measured at two different facets and are averaged. The inset shows a typical optical microscope picture. Conditions: $5.0 \mathrm{M} \mathrm{KOH}$ at $48.0^{\circ} \mathrm{C}$.

the measured current on surface area (compare Fig. 2 and 3). With the flow cell it is possible to observe the sample and measure dimensions directly during etching. Due to the sample geometry (see inset of Fig. 2), only the (100) facet remains parallel to the focal plane of the optical microscope. The (111) sidewalls of the V-grooves are at an angle to the surface of the sample, and they appear dark as they do not reflect light into the objective lens of the microscope. Due to this contrast, the width of the (100) facet $\left(\mathrm{L}_{(100)}\right)$ could be determined accurately. Figure 4 shows an example of the results of such an experiment, in which $\mathrm{L}_{(100)}$ is measured during the formation of $\mathrm{V}$-grooves. The inset of the graph shows a typical optical microscope picture, in which the (100) facet appears bright and the (111) facet dark. The figure shows that the width of the (100) facet, i.e., its surface area, decreases linearly with time, as was also observed with SEM (see Fig. 2) and peak current measurements (see Fig. 3a and b).

An application.-Various groups have shown that the chemical etch rate of silicon in alkaline solution can be influenced by applying an electrochemical potential to the etching wafer. The most extensive work was carried out by Xia et al. ${ }^{25}$ For these experiments, $\mathrm{n}$ - and p-type silicon (100) was etched with an O-ring as mask. After etching for a given time at fixed potential the etched depth was measured with a profilometer.

Using our electrochemical approach we have reproduced the trends found in Xia's work. ${ }^{25}$ Anisotropic etching of n-type and p-type Si was carried out a range of fixed applied potentials. Etching was interrupted at regular intervals during V-groove formation, and a number of voltammograms were measured at each potential. From the evolution of the (100) peak current the etch rate at a given potential could be determined. In Fig. 5a and $b$ these etch rates are plotted as a function of the applied potential for p-type and n-type silicon, respectively. In both cases, the etch rate decreases markedly as the surface becomes anodically passivated at positive potentials. Under cathodic polarization, the etch rate of p-type Si is essentially constant (see Fig. 5a) whereas in the case of n-type the etch rate drops considerably on going to more negative potentials (see Fig. $5 b)$. The reasons for such trends have been discussed elsewhere. ${ }^{25}$

\section{Conclusions}

Due to the geometry of the V-groove used in this work, the width of the (100) facet decreases continuously during anisotropic dissolution at a rate determined by the (100) etch rate. With electrochemical measurements or optical microscopic observation of the groove, 

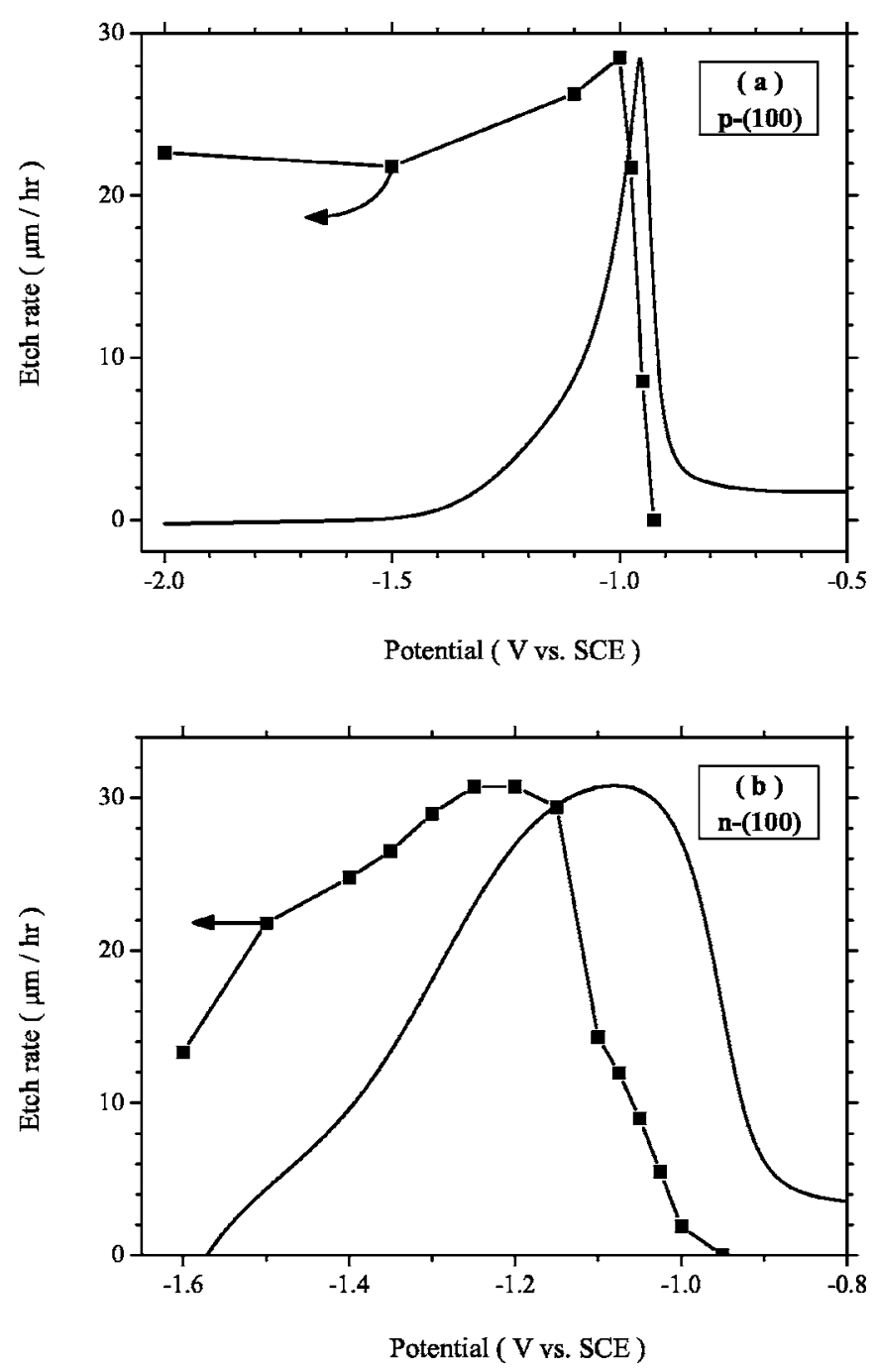

Figure 5. Etch rate as a function of applied potential for (a) p-type and (b) n-type $\mathrm{Si}(100)$ in $5.0 \mathrm{M} \mathrm{KOH}$ at $60.0^{\circ} \mathrm{C}$. Data points are connected to guide the eye. Measurements are based on the difference in (100) peak currents in voltammograms measured before and after polarization. The calculated etch rate was calibrated with the etch rate at open-circuit (see Fig. 2). The potential was applied for $30 \mathrm{~min}$. Normalized voltammograms are shown for comparison with the etch rate data points.

the etch rate can be measured in situ. In this way, the influence of external parameters such as potential or additives can be easily studied. These in situ approaches may also be applicable to other $\mathrm{Si}$ faces [the (110), for example] or to the anisotropic etching of other semiconductors such as GaAs and $\mathrm{InP}^{26}$

The electrochemical method described in this paper might also be used for process control in the batch fabrication of devices. A reference wafer with an Ohmic contact and an external source could be used to continuously monitor the etching system.
In the present work V-groove formation is considered. However, the electrochemical method can be used to monitor and determine the end point of formation of various geometries. Deep trenches ${ }^{27}$ and structures etched in the bulk of a wafer are all poorly accessible with optical microscopy and SEM without cleaving the material. In that case, it is advantageous to have a simple method to monitor the etching process. D'Arrigo et al. have shown that it is possible by measuring voltammograms to study the formation of rhomboidal microchannels in the bulk of a (110) wafer. ${ }^{28}$

\section{Acknowledgments}

The authors thank Duy Nguyen $\left(\mathrm{MESA}^{+}\right.$Research Institute, University of Twente, The Netherlands), Ismail Shah (Radboud University Nijmegen, The Netherlands) for valuable discussions and Jerôme Oskam for his help with part of the measurements. This work was financially supported by the Dutch Technology Foundation (STW, TPC-5990)

Utrecht University assisted in meeting the publication costs of this article.

\section{References}

1. M. Elwenspoek and H. Jansen, in Silicon Micromachining, Vol. 7, Cambridge University, Cambridge (1998)

2. R. M. Tiggelaar, T. T. Veenstra, R. G. P. Sanders, J. G. E. Gardeniers, M. C. Elwenspoek, and A. van den Berg, Talanta, 56, 331 (2002).

3. M. Hoffmann and E. Voges, J. Micromech. Microeng., 12, 349 (2002).

4. R. E. Oosterbroek, J. W. Berenschot, H. V. Jansen, A. J. Nijdam, G. Pandraud, A van den Berg, and M. C. Elwenspoek, J. Microelectromech. Syst., 9, 390 (2000).

5. B. Wacogne, Z. Sadani, and T. Gharbi, Sens. Actuators, A, 112, 328 (2004).

6. H. Seidel, L. Csepregi, A. Heuberger, and H. Baumgärtel, J. Electrochem. Soc., 137, 3612 (1990)

7. R. A. Wind and M. A. Hines, Surf. Sci., 460, 21 (2000).

8. Q. D. Nguyen and M. Elwenspoek, in Proceedings of the 16th Micromechanics Europe (MME) Workshop, p. 45 (2005).

9. K. Sato, M. Shikida, T. Yamashiro, K. Asaumi, Y. Iriye, and M. Yamamoto, Sens. Actuators, A, 73, 131 (1999)

10. E. van Veenendaal, K. Sato, M. Shikida, and J. van Suchtelen, Sens. Actuators, A, 93, 219 (2001)

11. P. J. Hesketh, C. Ju, S. Gowda, E. Zanoria, and S. Danyluk, J. Electrochem. Soc., 140, 1080 (1993)

12. H.-R. Kretschmer, X. H. Xia, J. J. Kelly, and A. Steckenborn, J. Electrochem. Soc., 151, C633 (2004)

13. A. J. Nijdam, J. G. E. Gardeniers, J. W. Berenschot, E. van Veenendaal, J. van Suchtelen, and M. Elwenspoek, J. Micromech. Microeng., 11, 499 (2001).

14. S. Tan, R. Boudreau, and M. L. Reed, Sens. Mater, 13, 303 (2001).

15. O. J. Glembocki, R. E. Stahlbusch, and M. Tomkiewicz, J. Electrochem. Soc., 132 145 (1985).

16. H. Tosaka, K Minami, and M. Esashi, J. Micromech Microeng, 5, 41 (1995).

17. E. Steinsland, T. Finstad, and A. Hanneborg, J. Electrochem. Soc, 146, 3890 (1999).

18. S. Tan, R. Boudreau, and M. L. Reed, Sens. Mater, 15, 101 (2003).

19. H. G. G. Philipsen and J. J. Kelly, J. Phys. Chem. B, 109, 17245 (2005).

20. R. L. Smith, B. Kloeck, N. de Rooij, and S. D. Collins, J. Electroanal. Chem. Interfacial Electrochem., 238, 103 (1987).

21. A. S. Louro and J. R. Senna, Proc. SPIE, 4557, 261 (2001)

22. J. W. Berenschot, R. E. Oosterbroek, T. S. J. Lammerink, and M. C. Elwenspoek, J. Micromech. Microeng., 8, 104 (1998).

23. M. Vangbo and Y. Bäcklund, J. Micromech. Microeng., 6, 279 (1996).

24. X. Xia, C. M. A. Ashruf, P. J. French, J. Rappich, and J. J. Kelly, J. Phys. Chem. $B, \mathbf{1 0 5}, 5722$ (2001).

25. X. H. Xia and J. J. Kelly, Phys. Chem. Chem. Phys., 3, 5304 (2001).

26. J. Wang, D. A. Thompson, and J. G. Simmons, J. Electrochem. Soc., 145, 2931 (1998).

27. A. Hölke and H. T. Henderson, J. Micromech. Microeng., 9, 51 (1999).

28. G. D'Arrigo, C. Spinella, E. Rimini, L. Rubino, and S. Lorenti, J. Electrochem. Soc., 151, C523 (2004). 